\title{
Brownian motion and exposed solutions of differential inclusions
}

\author{
Giovanni Colombo and Vladimir V. Goncharov
}

\begin{abstract}
We present a research program designed by A. Bressan and some partial results related to it. First, we construct a probability measure supported on the space of solutions to a planar differential inclusion, where the right-hand side is a Lipschitz continuous segment. Such measure assigns probability one to solutions having derivatives a.e. equal to one of the endpoints of the segment. Second, for a class of planar differential inclusions with Hölder continuous right-hand side $F$, we prove existence of solutions whose derivatives are exposed points of $F$. Finally, we complete the research program if the right-hand side of the differential inclusion does not depend on the state and prove a result on the Lipschitz continuity of an auxiliary map. The proofs rely on basic properties of Brownian motion.
\end{abstract}

Mathematics Subject Classification. 34A60, 60J65

\section{Introduction}

Let $F$ be a compact convex valued map from $\mathbb{R}^{N}$ into $\mathbb{R}^{N}$ and consider the differential inclusion

$$
\dot{x} \in F(x)
$$

Dedicated to Arrigo Cellina on the occasion of his 70th birthday.

The authors are supported by M.I.U.R. project "Viscosity, metric, and control theoretic methods for nonlinear partial differential equations", GNAMPA of INDAM, Fondazione CaRiPaRo Project "Nonlinear Partial Differential Equations: models, analysis, and control-theoretic problems" and University of Padova research project "Some analytic and differential geometric aspects in Nonlinear Control Theory, with applications to Mechanics", the project VARIANT PTDC/MAT/111809/2009 "Variational Analysis and Applications", funded by the Portuguese institutions FCT, COMPETE, QREN, and the European Regional Development Fund (FEDER). 
In his early pioneering paper [4], Cellina noted, in a particular case, that solutions whose derivatives belong to the set of extremal points of $F(x)$ (labeled as "extremal solutions" in this paper) are rather special, in the sense that they form a dense $G_{\delta}$ set in the set of solutions of (1.1) (a detailed discussion of the conditions under which this statement holds can be found in the survey paper [13]). Taking some inspiration from [4], F.S. De Blasi and G. Pianigiani designed a new method for proving existence of extremal solutions to differential inclusions based on a Baire category argument. A thorough account of their approach is contained in [13] (see also references therein, the further survey paper [7], and the most recent paper [8]). Here we only describe it briefly.

Trying to prove existence of solutions for differential inclusions via usual discretization methods, one immediately encounters the problem of pointwise convergence of derivatives of the approximations (see, e.g., [5]). Designing an algorithm ensuring such convergence is sometimes possible, as for example in A.F. Filippov's original proof of existence for differential inclusions with continuous compact valued right-hand side (see [9] or Theorem 1, p. 112, in [1]), but it is certainly useless if the right-hand side does not have closed values, as in the case of extremal points. The Baire category method, instead, does not require any such algorithm. Rather, the set of extremal solutions is proved to be the intersection of a sequence of open and dense subsets of a suitable complete metric space (the set of solutions of the convexified differential inclusion), and so by Baire's theorem it is dense. In this way, the nonemptiness of a certain set, namely the set of extremal solutions, follows from showing the much stronger fact that it is "large" in the sense of Baire category. This method has proved to be very effective, even in Banach spaces, in order to establish the existence of solutions to certain classes of nonconvex and nonclosed differential inclusions. In particular, it is possible to give another proof of Filippov's theorem (see [3]).

The present paper tries to consider the same problem from a probability point of view rather than from a metric one. Immediately after the Introduction, Sect. 2 contains, nearly in its original form, a research program designed by A. Bressan and proposed to the first author several years ago. Its aim is constructing a somewhat canonical probability measure in the space $\mathcal{C}\left([0, T] ; \mathbb{R}^{N}\right)$, which is supported exactly on the solution set of (1.1) and furthermore assigns probability one to the set of all extremal solutions. In other words, the same viewpoint of the Baire category method is taken, with a probabilistic idea of "large" set in place of a topological one. The proposed construction is based on the (uniformly distributed) Brownian motion on the unit sphere $S^{N-1}$ of $\mathbb{R}^{N}$ by exploiting essentially the following considerations:

1. for any Brownian path $\omega(\cdot)$ and any $x$, the unit vector $\omega(t)$ selects an extremal face of $F(x)$ by normality (i.e., by maximization of a scalar product);

2. for every $x$, normals to extremal faces of $F(x)$ which are not singletons form a zero measure subset of $S^{N-1}$ and furthermore, along a solution $x(\cdot)$ of (1.1), such normals should evolve with respect to time "much slower" than the a.s. Brownian path. As a result, with probability one a 
solution $x(\cdot)$ of (1.1) whose derivative at a.e. time $t$ maximizes the scalar product with $\omega(t)$ among all vectors of $F(x(t))$ should not be orthogonal to a face of $F(x(t))$. Thus it should be for a.e. $t$ an extremal point of $F(x(t))$, and actually an exposed point, as it will be explained later.

In this paper (see Sect. 5) we carry out the research program in the very special case where $F(x)$ is a segment in $\mathbb{R}^{2}$, Lipschitz continuous with respect to $x$ (the outline of the argument is again due to A. Bressan). Furthermore (Sect. 6), still remaining in a two dimensional setting, we prove that with probability one the solutions constructed by the method described in Sect. 2 are actually exposed solutions. Here the right-hand side of (1.1) is a general convex and compact set, Hölder continuous with respect to $x$, with exponent $\alpha>\frac{1}{2}$. This result can therefore be seen as a new existence result for (two dimensional) differential inclusions, as extremal points are not necessarily exposed. The program outlined in Sect. 2 is not completed in this case, since (under the Lipschitz assumption) we are not able to prove the conjectured a.s. uniqueness of exposed solutions associated with Brownian paths. The probabilistic facts on which the results are based are proved in Sect. 4 . Finally, we present some remarks concerning the general case. In particular, the conjectures in Sect. 2 can be fully proved in $\mathbb{R}^{N}$ if the right-hand side of (2.1) is independent of $x$. As an appendix (Sect. 8) we prove regularity of the auxiliary mapping used in Section 2.

\section{Random paths of differential inclusions}

Let $x \mapsto F(x) \subset \mathbb{R}^{N}$ be a Lipschitz continuous multifunction with compact convex values. Consider the Cauchy problem

$$
\begin{aligned}
& \dot{x}(t) \in F(x(t)), \quad t \in[0, T], \\
& x(0)=\bar{x} .
\end{aligned}
$$

We wish to put a "canonical" probability measure $\mu$ on the space of continuous functions $\mathcal{C}\left([0, T] ; \mathbb{R}^{N}\right)$ which is supported on the set $\mathcal{S}$ of trajectories of (2.1)-(2.2). Moreover, calling $\mathcal{S}^{\text {ext }}$ the set of trajectories of

$$
\dot{x}(t) \in \operatorname{ext} F(x)
$$

with initial data $(2.2),{ }^{1}$ the following properties should hold:

$$
\begin{aligned}
& \text { for every open set } U \subset \mathcal{C}\left([0, T] ; \mathbb{R}^{N}\right), \\
& \qquad \begin{array}{c}
U \cap \mathcal{S} \neq \emptyset \Longleftrightarrow \mu(U)>0 ; \\
\mu\left(\mathcal{S}^{\text {ext }}\right)=1 .
\end{array}
\end{aligned}
$$

The following construction represents an attempt to achieve this goal. Let $\omega$ denote Brownian motion on the surface of the unit sphere $S^{N-1} \subset \mathbb{R}^{N}$, with initial data uniformly distributed on $S^{N-1}$. Of course, by symmetry this

\footnotetext{
${ }^{1}$ ext $K$ denotes the set of extremal point of a convex set $K$.
} 
implies that the random variable $\omega(t)$ is still uniformly distributed on $S^{N-1}$ for every $t>0$. The space $\Omega$ of all such Brownian paths will be our basic probability space.

For each unit vector $p$ we define the compact, convex set

$$
F^{p}(x)=\left\{y \in F(x): y \cdot p=\max _{y^{\prime} \in F(x)} y^{\prime} \cdot p\right\} .
$$

Given a sample path $t \mapsto \omega(t)$, we want to construct a solution $t \mapsto x^{\omega}(t)$ of the reduced differential inclusion

$$
\dot{x}^{\omega}(t) \in F^{\omega(t)}(x(t)) .
$$

Such solution certainly exists. We conjecture that it is unique, for almost every $\omega \in \Omega$. To construct $x^{\omega}(\cdot)$, one may proceed as follows. Define $\beta(K)$ as the barycenter of the ball $B(K, 1)$ of radius 1 around the set $K$ and set

$$
F_{m}(x, p):=\left\{y \in F(x): p \cdot y+\frac{1}{m} \geq \max _{y^{\prime} \in F(x)} p \cdot y^{\prime}\right\} .
$$

For $m \geq 1$ and each sample path $\omega$, define $x_{m}^{\omega}$ as the unique solution to the Cauchy problem ${ }^{2}$ :

$$
\dot{x}(t)=\beta\left(F_{m}(x(t), \omega(t))\right), \quad x(0)=\bar{x} .
$$

Letting $m \rightarrow \infty$, we guess that both

$$
x_{m}^{\omega}(t) \rightarrow x^{\omega}(t) \quad \text { uniformly on }[0, T],
$$

and

$$
\dot{x}^{\omega}(t) \in \operatorname{ext} F\left(x^{\omega}(t)\right)
$$

hold for a.e. sample path $\omega \in \Omega$.

\section{Technical preliminaries}

We will denote by $|E|$ the Lebesgue measure of a set $E \subset \mathbb{R}$, by $\mathcal{H}^{d}$ the $d$ dimensional Hausdorff measure, and by $1_{E}$ the characteristic function of $E$, i.e., $1_{E}(t)=1$ if $t \in E, 1_{E}(t)=0$ if $t \notin E$. The Hausdorff distance between subsets of $\mathbb{R}^{N}$ will be denoted by $H(\cdot, \cdot)$, and the interior of a set $A$ is int $A$. The distance from a set $K$ is denoted by $d_{K}(x)=\inf \{\|x-y\|: y \in K\}$. It is well known that if $K$ is a closed and convex subset of $\mathbb{R}^{N}$ then $d_{K} \in \mathcal{C}^{1}\left(\mathbb{R}^{N} \backslash K\right)$. Moreover,

$$
\nabla d_{K}(x)=\left(x-\pi_{K}(x)\right) / d_{K}(x) \quad \forall x \notin K,
$$

where $\pi_{K}(x)$ is the point in $K$ closest to $x$, and $\nabla d_{K}(x)$ is normal to $K$ at $\pi_{K}(x)$, i.e., $\left\langle\nabla d_{K}(x), y-\pi_{K}(x)\right\rangle \leq 0$ for all $y \in K$.

\footnotetext{
${ }^{2}$ We recall (see $[1$, Theorem 1, p. 77]) that the barycentric selection from a Lipschitz setvalued map is Lipschitz. The Lipschitz continuity of $F_{m}$ will be proved in Sect. 8. Hence, the uniqueness of the solution to (2.7) follows.
} 
Let $K \subset \mathbb{R}^{N}$ be closed and convex and let $x \in K$. We say that $x$ is an exposed point of $K$ if there exists a support hyperplane to $K$ which touches $K$ only at $x$. Equivalently, $x$ is an exposed point of $K$ if there exists a vector $\zeta$ such that

$$
\zeta \cdot(y-x)<0 \quad \forall y \in K, y \neq x .
$$

We denote by $\exp K$ the set of exposed points of $K$. It is well known that $\exp K$ is nonempty for every compact and convex $K \subset \mathbb{R}^{N}$.

Remark. Let $K$ be the convex hull of the two closed circles $B((-1,0), 1)$ and $B((1,0), 1)$ in $\mathbb{R}^{2}$. The points $(-1, \pm 1)$ and $(1, \pm 1)$ are extremal points of $K$ (i.e., they do not belong to the relative interior of any segment contained in $K)$, but are not exposed. In particular, the set of exposed points of $K$ is not closed.

The support function to a convex closed set $K$ is defined as

$$
\sigma_{K}(p)=\max _{v \in K} p \cdot v, \quad p \in \mathbb{R}^{N} .
$$

Observe that

$$
\left\{v \in K: p \cdot v=\sigma_{K}(p)\right\}=\partial \sigma_{K}(p),
$$

where $\partial \sigma_{K}(p)$ is the subdifferential in the sense of Convex Analysis.

The following fact reflects the isometry between the space of convex compact subsets of $\mathbb{R}^{N}$ and a cone in the space of continuous functions. Although it is well known, we give a short proof for the sake of completeness.

Lemma 3.1. Let $A, B$ be compact and convex in $\mathbb{R}^{N}$. Then

$$
H(A, B)=\sup _{p \in S^{N-1}}\left|\sigma_{A}(p)-\sigma_{B}(p)\right| .
$$

Proof. Let $x \in A$. If $x \notin B$, set $p=\left(x-\pi_{B}(x)\right) / d(x, B)$. Then clearly $p$. $\pi_{B}(x)=\sigma_{B}(p)$, so that $\left|\sigma_{A}(p)-\sigma_{B}(p)\right| \geq p \cdot x-p \cdot \pi_{B}(x)=d(x, B)$. The converse inequality is easy.

For further definitions and results on set-valued analysis and differential inclusions we refer to Chapters 1 and 2 in [1], while for basic definitions and properties on stochastic processes and the scalar Brownian motion we refer to Chapters 1 and 2 in [11].

We will denote by $(\Omega, \omega(t), \mathcal{F}, P)$ a standard one dimensional Brownian motion. In what follows we will consider also a real random variable $Y$, uniformly distributed on the interval $[0,2 \pi]$, independent of $\mathcal{F}$. We consider the stochastic process $\Theta=([0,2 \pi] \times \Omega, \theta(t), \mathcal{L} \otimes \mathcal{F}, Q \otimes P)$, where $\mathcal{L}$ denotes the $\sigma$-algebra of Lebesgue measurable subsets of $[0,2 \pi], Q$ denotes the normalized Lebesgue measure on $[0,2 \pi]$, and for each $y \in[0,2 \pi]$ and $\omega \in \Omega, \theta=\theta^{y, \omega}(t)$ is the unique angle in $[0,2 \pi)$ such that $e^{i \theta(t)}=e^{i(y+\omega(t))}$. The distance between two such angles $\theta_{1}$ and $\theta_{2}$, i.e., the length of the corresponding arc in the unit circle, will be denoted by $\rho\left(\theta_{1}, \theta_{2}\right)$.

Proposition 3.2. For all $t \geq 0$, the random variable $\theta(t)$ is uniformly distributed in $[0,2 \pi]$. 
Proof. Let $\Phi: \mathbb{R} \rightarrow \mathbb{R}$ be $2 \pi$-periodic, continuous, and bounded. We have

$$
\begin{aligned}
\int_{[0,2 \pi] \times \Omega} \Phi(Y+\omega(t)) d Q \otimes d P & =\int_{0}^{2 \pi} \int_{-\infty}^{+\infty} \Phi(y+x) \frac{e^{-\frac{x^{2}}{2 t}}}{\sqrt{2 \pi t}} d x \frac{d y}{2 \pi} \\
& =\int_{0}^{2 \pi} \int_{-\infty}^{+\infty} \Phi(u) \frac{e^{-\frac{(u-y)^{2}}{2 t}}}{\sqrt{2 \pi t}} d y \frac{d u}{2 \pi} \\
& =\int_{0}^{2 \pi} \frac{\Phi(u)}{2 \pi} d u .
\end{aligned}
$$

\section{Brownian paths and continuous functions}

In order to proceed with the proofs we need two results on Brownian paths, to which this section is devoted. In this section, $I=[0, T]$ is an interval and $(\Omega, P)$ is a standard Brownian motion.

The first result is concerned with the fact that a.s. a Brownian path cannot be Hölder with exponent $\alpha>1 / 2$ on a set of positive measure (dependent on $\omega$ ).

We are given a function $\varphi: I \times \Omega \rightarrow \mathbb{R}$, which is assumed to be measurable in $\omega$ for all $t \in I$, and Hölder in $t$ for a.e. $\omega \in \Omega$ (with the same exponent $\alpha>\frac{1}{2}$ and the same constant $L>0)$. We denote by $A:=\{(t, \omega) \in I \times \Omega: \omega(t)=$ $\varphi(t, \omega)\}$ and by $A_{t}:=\{\omega \in \Omega: \omega(t)=\varphi(t, \omega)\}, A^{\omega}:=\{t \in I: \omega(t)=\varphi(t, \omega)\}$ the sections of the set $A$.

The following is the main result on this point.

Theorem 4.1. Under the assumptions above, P-almost surely the set $A^{\omega}$ is negligible w.r.t. the Lebesgue measure. More precisely, given $\eta>0$ there exists at most a finite set of points $J \subset I$ such that $P\left(A_{t}\right) \geq \eta$ for $t \in J$.

The proof requires the following lemma:

Lemma 4.2. For each finite number of points $0 \leq t_{1}<t_{2}<t_{3}<\cdots<t_{n} \leq T$ the inequality

$$
P\left[\bigcup_{i=1}^{n} A_{t_{i}}\right] \geq \sum_{i=1}^{n} P\left(A_{t_{i}}\right)-\frac{L}{\sqrt{2 \pi}} h^{\alpha-\frac{1}{2}} n(n-1),
$$

where $h:=\max \left\{\left|t_{i}-t_{j}\right|: 1 \leq i, j \leq n\right\}$, holds.

Proof. We prove this statement by induction w.r.t. $n=1,2, \ldots$. For $n=1$ it is obvious. Assume now that for a given $n$ the inequality (4.1) holds. Then for $n+1$ points we have:

$$
P\left[\bigcup_{i=1}^{n+1} A_{t_{i}}\right]=P\left[\bigcup_{i=1}^{n} A_{t_{i}}\right]+P\left(A_{t_{n+1}}\right)-P\left[\left(\bigcup_{i=1}^{n} A_{t_{i}}\right) \cap A_{t_{n+1}}\right] .
$$


Applying now the induction hypothesis we obtain from (4.2)

$$
\begin{aligned}
P\left[\bigcup_{i=1}^{n+1} A_{t_{i}}\right] \geq & \sum_{i=1}^{n+1} P\left(A_{t_{i}}\right)-\frac{L}{\sqrt{2 \pi}} h^{\alpha-\frac{1}{2}} n(n-1) \\
& -P\left[\bigcup_{i=1}^{n}\left(A_{t_{i}} \cap A_{t_{n+1}}\right)\right] .
\end{aligned}
$$

Let us estimate each probability $P\left(A_{t_{i}} \cap A_{t_{n+1}}\right), i=1,2, \ldots, n$. By our definition we obviously have:

$$
A_{t_{i}} \cap A_{t_{n+1}} \subset\left\{\omega \in \Omega:\left|\omega\left(t_{n+1}\right)-\omega\left(t_{i}\right)\right| \leq L\left(t_{n+1}-t_{i}\right)^{\alpha}\right\} .
$$

Set $h_{i}:=t_{n+1}-t_{i}$. We have:

$$
\begin{aligned}
P\left(A_{t_{i}} \cap A_{t_{n+1}}\right) & \leq P\left\{\omega \in \Omega:-L h_{i}^{\alpha} \leq \omega\left(t_{i}+h_{i}\right)-\omega\left(t_{i}\right) \leq L h_{i}^{\alpha}\right\} \\
& =\frac{1}{\sqrt{2 \pi h_{i}}} \int_{-L h_{i}^{\alpha}}^{L h_{i}^{\alpha}} \exp \left(-\frac{x^{2}}{2 h_{i}}\right) d x \\
& =\frac{1}{\sqrt{2 \pi}} \int_{-L h_{i}^{\alpha-\frac{1}{2}}}^{L h_{i}^{\alpha-\frac{1}{2}}} \exp \left(-\frac{s^{2}}{2}\right) d s \\
& \leq L \sqrt{\frac{2}{\pi}}\left(h^{\prime}\right)^{\alpha-\frac{1}{2}}
\end{aligned}
$$

where $h^{\prime}:=\max \left\{\left|t_{i}-t_{j}\right|: 1 \leq i, j \leq n+1\right\}$. Then by semiadditivity of the probability we conclude from (4.4):

$$
P\left[\bigcup_{i=1}^{n}\left(A_{t_{i}} \cap A_{t_{n+1}}\right)\right] \leq L \sqrt{\frac{2}{\pi}}\left(h^{\prime}\right)^{\alpha-\frac{1}{2}} n .
$$

From (4.3) it follows now that

$$
\begin{aligned}
P\left[\bigcup_{i=1}^{n+1} A_{t_{i}}\right] & \geq \sum_{i=1}^{n+1} P\left(A_{t_{i}}\right)-\frac{L}{\sqrt{2 \pi}}\left(h^{\prime}\right)^{\alpha-\frac{1}{2}} n(n-1)-L \frac{1}{\sqrt{2 \pi}}\left(h^{\prime}\right)^{\alpha-\frac{1}{2}} \cdot 2 n \\
& =\sum_{i=1}^{n+1} P\left(A_{t_{i}}\right)-\frac{L}{\sqrt{2 \pi}}\left(h^{\prime}\right)^{\alpha-\frac{1}{2}}(n+1) n,
\end{aligned}
$$

and the lemma is proved.

We are now ready for the proof of Theorem 4.1 .

Proof. Let us assume by contradiction that there exists a sequence of different points $\left\{t_{1}, t_{2}, \ldots, t_{n}, \ldots\right\} \subset I$ such that $P\left(A_{t_{i}}\right) \geq \eta, i=1,2, \ldots$. Since $I$ is a compact interval, the sequence $\left\{t_{n}\right\}$ admits a cluster point $\tau \in I$. Without loss of generality (taking a subsequence if necessary) we can assume that $\left\{t_{n}\right\}$ is strictly monotone (say, increasing) and converges to $\tau$. Since $\left\{t_{n}\right\}$ is a Cauchy sequence, given $N=1,2, \ldots$ we can choose $k(N)$ such that

$$
\left|t_{m}-t_{n}\right| \leq\left(\frac{\eta^{2} \pi}{2 L^{2}} \cdot \frac{1}{N^{2}}\right)^{\frac{1}{2 \alpha-1}}
$$


whenever $m, n \geq k(N)$. Let us consider the following family of sets

$$
\left\{A_{t_{k(N)}}, A_{t_{k(N)+1}}, A_{t_{k(N)+2}}, \ldots, A_{t_{k(N)+N}}\right\}
$$

and apply to them Lemma 4.2. We have here $N+1$ sets, and the probability of each of them is $\geq \eta$. Furthermore (see (4.5)),

$$
\begin{aligned}
h:=\max \left\{\left|t_{i}-t_{j}\right|: i, j=k(N), k(N)\right. & +1, \ldots, k(N)+N\} \\
\leq & \left(\frac{\eta^{2} \pi}{2 L^{2}} \cdot \frac{1}{N^{2}}\right)^{\frac{1}{2 \alpha-1}} .
\end{aligned}
$$

Thus, we obtain from (4.1):

$$
P\left[\bigcup_{i=0}^{N} A_{t_{k(N)+i}}\right] \geq(N+1) \eta-\frac{L}{\sqrt{2 \pi}} \cdot \frac{\eta \sqrt{\pi}}{\sqrt{2} L N} N(N+1)=\frac{1}{2}(N+1) \eta,
$$

and this is a contradiction because $N=1,2, \ldots$ can be chosen as large as we want.

Now the first statement of Theorem 1 follows directly from the above remark and Fubini's theorem.

The second result of the section is concerned with the probability of a Brownian path to remain close to a given continuous function. Although it is well known, we present a proof for the sake of completeness.

Proposition 4.3. Let $\varphi: I \rightarrow[0,2 \pi]$ be continuous. Let $\varepsilon>0$ and $y$ be such that $\rho(y, \varphi(0))<\varepsilon / 2$. Then

$$
P\{\omega: \rho(y+\omega(t), \varphi(t))<\varepsilon \quad \forall t \in I\}>0 .
$$

Proof. Let

$$
w(\delta)=\sup _{s, t \in I,|t-s| \leq \delta}|\varphi(t)-\varphi(s)|
$$

and set

$$
\eta(\delta)=\max \left\{w(\delta), 2 \sqrt{\delta \log \log \frac{1}{\delta}}\right\} .
$$

Let $\bar{\delta}$ be such that $0<\delta<\bar{\delta}$ implies $\eta(\delta)<\varepsilon / 2$. If $0<\delta<\bar{\delta}$ is small enough, then, by the Law of the Iterated Logarithm (see, e.g., Theorem 2.9.23 in [11]) and the distribution of $\omega(\delta)$ we have

$$
P\left\{\omega: \rho(y+\omega(t), \varphi(t))<\varepsilon \forall t \in[0, \delta], \rho(y+\omega(\delta), \varphi(\delta))<\frac{\varepsilon}{2}\right\}>0 .
$$

Since, $\omega(\delta+t)-\omega(\delta)$ is still a Brownian motion, we can repeat the above procedure $n$ times, until $n \delta>T$. 


\section{A special case}

This section is devoted to the case where

$$
F(x)=\operatorname{co}\{f(x), g(x)\} \subset \mathbb{R}^{2}
$$

with $f, g$ Lipschitz vector fields with Lipschitz constant $L$, such that $f(x) \neq$ $g(x)$ for every $x \in \mathbb{R}^{2}$. Without loss of generality, we assume also that $\|f(x)\|$, $\|g(x)\| \leq M$ for all $x \in \mathbb{R}^{2}$.

Let $T>0$ be fixed. Given the process $\Theta$ defined in Sect. 3, let $t \mapsto$ $x^{\theta}(t)\left(=x^{y, \omega}(t)\right)$ be the solution to the Cauchy problem

$$
\dot{x}(t)=\left\{\begin{array}{l}
f(x(t)) \text { if } \cos \theta^{y, \omega}(t)>0, \\
g(x(t)) \text { if } \cos \theta^{y, \omega}(t)<0,
\end{array} \quad x(0)=\bar{x} .\right.
$$

By standard properties of Brownian motion, with probability one we have

$$
\cos \theta^{y, \omega}(t) \neq 0 \quad \text { for a.e. } t \in[0, T] .
$$

Therefore the solution of (5.2) is a.s. well defined. Moreover,

Proposition 5.1. With probability one the Cauchy problem (5.2) admits a unique solution on $[0, T]$.

Proof. Fix $\theta(\cdot)$ such that (5.3) holds. Then, for a.e. $t \in[0, T]$ the right-hand side of (5.2) is Lipschitz with respect to $x$, and for all $x$ it is measurable with respect to $t$. Then the statement follows from the measurable version of Picard-Lindelöf theorem (see, e.g., [16, p. 121]).

For every $x \in \mathbb{R}^{2}$ consider the angle $\alpha(x)$ between the vector $f(x)-g(x)$ and the positive $x_{1}$-axis (defined up to multiples of $2 \pi$ ). For every $(y, \omega) \in[0,2 \pi) \times \Omega$ the map

$$
t \mapsto \alpha^{y, \omega}(t) \doteq \alpha\left(x^{y, \omega}(t)\right)
$$

is Lipschitz continuous. For each fixed $y \in[0,2 \pi)$, we are now going to construct a new probability measure $\tilde{P}_{y}$ on $\Omega$ such that the push-forward of $\tilde{P}_{y}$ w.r.t. the map

$$
\omega(t) \mapsto \omega(t)+\alpha^{y, \omega}(t)
$$

is Wiener measure (i.e., standard Brownian motion).

Proposition 5.2. For each fixed $y \in[0,2 \pi]$ there exists a probability measure $\tilde{P}_{y}$ on $\Omega$ absolutely continuous with respect to $P$ such that

$$
\frac{d \tilde{P}_{y}}{d P}=\exp \left[-\int_{0}^{t} \dot{\alpha}^{y, \omega}(s) d \omega(s)-\frac{1}{2} \int_{0}^{t}\left(\dot{\alpha}^{y, \omega}(s)\right)^{2} d s\right],
$$

and

$$
\left(\Omega, \omega(t)+\alpha^{y, \omega}(t), \mathcal{F}, \tilde{P}_{y}\right) \quad \text { is a Brownian motion. }
$$

Proof. We wish to apply the Cameron-Martin-Girsanov formula (see [11, Section 3.5]) to $\omega(t)+\alpha^{y, \omega}(t)$. To this aim, we need to prove that

1. $(t, \omega) \mapsto \dot{\alpha}^{y, \omega}(t)$ is jointly measurable and $\mathcal{F}_{t}$-adapted (we consider the natural filtration $\mathcal{F}_{t}$ ); 
2. the Novikov condition (see Corollary 5.13 in [11, Chapter 3]) holds, i.e.,

$$
E\left[\exp \left(\frac{1}{2} \int_{0}^{\tau}\left(\dot{\alpha}^{y, \omega}(s)\right)^{2} d s\right)\right]<+\infty \quad \text { for all } \tau \geq 0 .
$$

Observe that $(y, \omega) \mapsto x^{y, \omega}(t)$ is jointly measurable and $\mathcal{F}_{t}$-adapted, since $x^{y, \omega}$ is the solution of the differential equation (5.2). By Lipschitz continuity of $x^{y, \omega}$ (which is uniform with respect to $\omega$ and $y$ ), the (left) derivative $\dot{x}^{y, \omega}(t)$ is $P$-a.s. defined for a.e. $t \in[0, T]$. By setting $\dot{x}^{y, \omega}(t)=0$ for those $(t, \omega)$ where $\dot{x}^{y, \omega}(t)$ does not exist, we obtain a process which is jointly measurable, $\mathcal{F}_{t}$-adapted, and bounded. Hence conditions 1) and 2) are satisfied.

We define now a new probability measure on $[0,2 \pi) \times \Omega$ by setting, for all $0 \leq a \leq b<2 \pi$ and all $\mathcal{F}$-measurable set $B$

$$
\tilde{P}([a, b] \times B)=\int_{0}^{2 \pi} \tilde{P}_{y}(B) Q(d y)
$$

and a probability measure $\mu$ on $\mathcal{C}\left([0, T] ; \mathbb{R}^{2}\right)$ by setting, for every Borel set $A$,

$$
\mu(A)=\tilde{P}\left\{(y, \omega): x^{y, \omega} \in A\right\} .
$$

The definition is meaningful, since the single-valued map $(y, \omega) \mapsto x^{y, \omega}$ is measurable.

In order to show that the probability measure $\mu$ satisfies all the expected requirements, we need to prove the following:

Proposition 5.3. $1 . \operatorname{supp}(\mu)=\mathcal{S}$, i.e., for every open set $U \subset \mathcal{C}\left([0, T] ; \mathbb{R}^{2}\right)$,

$$
\mu(U)>0 \text { if and only if } U \cap \mathcal{S} \neq \emptyset,
$$

i.e., if and only if $U$ contains solutions of $\dot{x} \in \operatorname{co}\{f(x), g(x)\}, x(0)=\bar{x}$;

2. $\mu\{x \in \mathcal{S}: \dot{x}(t) \in\{f(x(t)), g(x(t))\}$ for a.e.t $\in[0, T]\}=1$.

Proof. Let $U \subset \mathcal{C}\left([0, T] ; \mathbb{R}^{2}\right)$ be open. If $U \cap \mathcal{S}=\emptyset$, then, obviously by construction, $\mu(U)=0$. Conversely, assume $U \cap \mathcal{S} \neq \emptyset$, fix $x \in U \cap \mathcal{S}$ and let $\varepsilon>0$ be such that

$$
\|y-x\|_{\infty}<\varepsilon \Rightarrow y \in U
$$

We wish to prove that

$$
\mu\left\{y: \dot{y} \in \operatorname{co}\{f(y), g(y)\} \text { a.e. and }\|y-x\|_{\infty}<\varepsilon\right\}>0 .
$$

For each $n=1,2, \ldots, i=0, \ldots, n-1$, set $t_{n}^{i}=\frac{i T}{n}$ and $I_{n}^{i}=\left[t_{n}^{i}, t_{n}^{i+1}\right)$. Let $\lambda:[0, T] \rightarrow[0,1]$ be measurable and such that, a.e. on $[0, T]$,

$$
\dot{x}(t)=\lambda(t) f(x(t))+(1-\lambda(t)) g(x(t)) .
$$

By a Corollary of Lyapunov's Convexity Theorem (see [12, Theorem 2]), for every $i=0, \ldots, n-1$, there exists a measurable subset $E_{n}^{i}$ of $I_{n}^{i}$ such that

$$
\begin{aligned}
\int_{E_{n}^{i}} f(x(t)) d t & =\int_{I_{n}^{i}} \lambda(t) f(x(t)) d t, \\
\int_{I_{n}^{i} \backslash E_{n}^{i}} g(x(t)) d t & =\int_{I_{n}^{i}}(1-\lambda(t)) g(x(t)) d t .
\end{aligned}
$$


Define

$$
\begin{aligned}
& u_{n}(t)=\sum_{i=1}^{n}\left[1_{E_{n}^{i}}(t) f(x(t))+1_{I_{n}^{i} \backslash E_{n}^{i}}(t) g(x(t))\right], \\
& x_{n}(t)=\bar{x}+\int_{0}^{t} u_{n}(s) d s .
\end{aligned}
$$

Observe that, by construction, $x_{n}\left(t_{n}^{i}\right)=x\left(t_{n}^{i}\right)$ for all $i=0, \ldots, n-1$. Therefore, for each $t \in I_{n}^{i}$,

$$
\left\|x(t)-x_{n}(t)\right\| \leq \int_{I_{n}^{i}}\left|1_{E_{n}^{i}}-\lambda(t)\right|(\|f(x(s))\|+\|g(x(s))\|) d s \leq \frac{2 T M}{n} .
$$

Moreover, since by construction $\dot{x}_{n}(t) \in\{f(x(t)), g(x(t))\}$ for a.e. $t \in[0, T]$, we have

$$
d\left(\dot{x}_{n}(t),\left\{f\left(x_{n}(t)\right), g\left(x_{n}(t)\right)\right\}\right) \leq L\left\|x_{n}(t)-x(t)\right\| \leq \frac{2 T L M}{n} .
$$

Therefore, by Filippov's theorem (see Theorem 2.4.1 in [1]) there exists a solution $y_{n}:[0, T] \rightarrow \mathbb{R}^{2}$ of the Cauchy problem

$$
\dot{x} \in\{f(x), g(x)\}, \quad x(0)=\bar{x}
$$

such that

$$
\left\|y_{n}-x_{n}\right\|_{\infty} \leq \frac{2 T M}{n}\left(e^{L T}-1\right)
$$

Let $A_{n} \subseteq[0, T]$ be such that $\dot{y}_{n}(t)=f\left(y_{n}(t)\right)$ for a.e. $t \in A_{n}$ and $\dot{y}_{n}(t)=$ $g\left(y_{n}(t)\right)$ for a.e. $t \in B_{n}:=[0, T] \backslash A_{n}$. Let $C_{n}$ (resp., $\left.K_{n}\right)$ be a closed subset of $A_{n}$ (resp., of $B_{n}$ ) such that $\left|A_{n} \backslash C_{n}\right|<\frac{1}{n}$ (resp., $\left|B_{n} \backslash K_{n}\right|<\frac{1}{n}$ ). Let $\varphi_{n}:[0, T] \rightarrow[0,2 \pi]$ be continuous and such that

$$
\begin{array}{ll}
\varphi_{n}(t)=0 & \text { for all } t \in C_{n}, \\
\varphi_{n}(t)=\pi & \text { for all } t \in K_{n} .
\end{array}
$$

For every sample path $\theta(\cdot)=\theta^{y, \omega}$ such that $\rho\left(\theta(t), \varphi_{n}(t)\right)<\frac{1}{n}$ for all $t \in[0, T]$ we have

$$
\begin{aligned}
\left\|x^{y, \omega}(t)-y_{n}(t)\right\| \leq & \int_{C_{n} \cap[0, t]}\left\|f\left(x^{y, \omega}(s)\right)-f\left(y_{n}(s)\right)\right\| d s \\
& +\int_{K_{n} \cap[0, t]}\left\|g\left(x^{y, \omega}(s)\right)-g\left(y_{n}(s)\right)\right\| d s \\
& +\int_{[0, t] \backslash\left(C_{n} \cup K_{n}\right)}\left(\left\|\dot{x}^{y, \omega}(s)\right\|+\left\|\dot{y}_{n}(s)\right\|\right) d s \\
\leq L & \int_{0}^{t}\left\|x^{y, \omega}(s)-y_{n}(s)\right\| d s+\frac{4 M}{n} .
\end{aligned}
$$

Therefore, by Gronwall's lemma

$$
\left\|x^{y, \omega}-y_{n}\right\|_{\infty} \leq \frac{4 M}{L}\left(e^{L T}-1\right) \frac{1}{n} .
$$


Thus, by putting together (5.8), (5.9), and (5.10) we obtain that for all $n \in \mathbb{N}$ large enough if $\theta^{y, \omega}$ satisfies the inequality $\rho\left(\theta^{y, \omega}(t), \varphi_{n}(t)\right)<\frac{1}{n}$ for all $t \in$ $[0, T]$, then

$$
\left\|x-x^{y, \omega}\right\|_{\infty}<\varepsilon .
$$

On the other hand, for all $n=1,2, \ldots$ we have

$$
\begin{aligned}
Q \otimes P & \left\{\theta: \rho\left(\theta(t), \varphi_{n}(t)\right)<\frac{1}{n} \quad \forall t \in[0, T]\right\} \\
& =\frac{1}{2 \pi} \int_{0}^{2 \pi} P\left\{\rho\left(\omega(t)+y, \varphi_{n}(t)\right)<\frac{1}{n} \forall t \in[0, T] \mid Y=y\right\} d y .
\end{aligned}
$$

Recalling Proposition 4.3, the integrand in the above expression is positive for a set of $y \in[0,2 \pi]$ with positive measure. Thus

$$
Q \otimes P\left\{\theta: \rho\left(\theta(t), \varphi_{n}(t)\right)<\frac{1}{n} \quad \forall t \in[0, T]\right\}>0 \quad \forall n=1,2 \ldots
$$

By Proposition 5.2,

$$
\tilde{P}\{\theta: \cos \theta(t) \neq 0 \text { a.e. }\}=1 \text {. }
$$

Now set $\tilde{\theta}(t)=\theta(t)+\alpha^{y, \omega}(t)$ and observe that the condition $\cos \theta(t)>0$ (resp., $\cos \theta(t)<0)$ means exactly that the angle between $(\cos \tilde{\theta}(t), \sin \tilde{\theta}(t))$ and $f\left(x^{y, \omega}(t)\right)-g\left(x^{y, \omega}(t)\right)$ belongs to the interval $(-\pi / 2, \pi / 2)$ (resp., to $(\pi / 2$, $3 \pi / 2))$. Therefore,

$$
\begin{array}{r}
\tilde{P}\left\{(y, \omega):(\cos \tilde{\theta}(t), \sin \tilde{\theta}(t)) \cdot \dot{x}^{y, \omega}(t)=\max \{(\cos \tilde{\theta}(t), \sin \tilde{\theta}(t)) \cdot v:\right. \\
\left.\left.v \in\left\{f\left(x^{y, \omega}(t)\right), g\left(x^{y, \omega}(t)\right)\right\}\right\} \text { a.e. in }[0, T]\right\}=1,
\end{array}
$$

whence, in particular,

$$
\tilde{P}\left\{(y, \omega): \dot{x}^{y, \omega}(t) \in\left\{f\left(x^{y, \omega}(t)\right), g\left(x^{y, \omega}(t)\right)\right\} \text { for a.e. } t \in[0, T]\right\}=1 .
$$

Consequently,

$$
\begin{aligned}
\mu\{x: \dot{x} \in\{f(x), & g(x)\} \text { a.e. } \operatorname{in}[0, T]\} \\
& =\tilde{P}\left\{(y, \omega): \dot{x}^{y, \omega}(t) \in\left\{f\left(x^{y, \omega}(t)\right), g\left(x^{y, \omega}(t)\right)\right\} \text { a.e. }\right\} \\
& =1
\end{aligned}
$$

and Part 2) is proved, by recalling that $\left(\Omega, \omega(t)+\alpha^{y, \omega}(t), \mathcal{F}, \tilde{P}_{y}\right)$ is a Brownian motion. In order to conclude the proof of Part 1), observe that we have

$$
\mu\left\{z: \dot{z} \in \operatorname{co}\{f(z), g(z)\} \text { a.e. and }\|z-x\|_{\infty}<\varepsilon\right\} \geq \tilde{P}\left\{\tilde{\theta}:\left\|\tilde{\theta}-\varphi_{n}\right\|_{\infty}<\frac{1}{n}\right\},
$$

and the right-hand side of the above inequality is positive thanks to Proposition 5.2 and (5.11). The proof is concluded.

Remarks. 1. In this case, the properties of the measure $\mu$ conjectured in Sect. 2 are fully satisfied. In fact, by construction the differential inclusion (2.5) has $\mu$-a.s. a unique solution. Thus the sequence constructed according to (2.7) is 
Cauchy (and actually is Cauchy in the strong topology of $W^{1,1}\left([0, T] ; \mathbb{R}^{2}\right)$, see the end of the proof of Theorem 6.1).

2. The argument using Girsanov's formula can be likely extended to Lipschitz polyhedra in the plane, provided angles between adjacent vertices with respect to a suitable internal point (e.g., the barycenter) remain unchanged. An extension to general Lipschitz polyhedra should instead require as many independent Brownian motions as couples of vertices.

\section{Exposed solutions in the plane}

Let $F$ be a compact and convex valued map from $\mathbb{R}^{2}$ into $\mathbb{R}^{2}$. We will assume that $F$ is Hölder continuous with exponent $\alpha>\frac{1}{2}$, i.e., there exists a constant $L$ such that

$$
H\left(F\left(x_{1}\right), F\left(x_{2}\right)\right) \leq L\left\|x_{1}-x_{2}\right\|^{\alpha} \quad \forall x_{1}, x_{2} \in \mathbb{R}^{2},
$$

where we recall that $H(\cdot, \cdot)$ denotes the Hausdorff distance between subsets of $\mathbb{R}^{2}$. Assume that there exists a constant $M$ such that

$$
\|v\| \leq M \quad \forall v \in F(x), \forall x \in \mathbb{R}^{2} .
$$

We will consider again the stochastic process $\Theta$ constructed in Sect. 3. For each sample path $\theta$ of $\Theta$ set

$$
\nu_{\theta}(t)=(\cos \theta(t), \sin \theta(t))
$$

and observe that a.s. $\nu_{\theta}$ is continuous. We note now that a.s. the differential inclusion

$$
\dot{x}^{\theta}(t) \in F^{\nu_{\theta}(t)}\left(x^{\theta}(t)\right), \quad x^{\theta}(0)=\bar{x}
$$

(recall (2.5)) admits solutions. Indeed, it is enough to observe that for a.e. $\theta$ and every $t$ the map $x \mapsto F^{\nu_{\theta}(t)}(x)$ has closed graph and compact convex values, and the same happens to $t \mapsto F^{\nu_{\theta}(t)}(x)$ for every $x$.

Our aim is to prove the following

Theorem 6.1. Under the above assumptions on $F$, for every $\bar{x} \in \mathbb{R}^{2}$ and for every $T>0$ the Cauchy problem

$$
\dot{x} \in \exp F(x), \quad x(0)=\bar{x}
$$

admits a Carathéodory solution in $[0, T]$. More precisely, with probability one, all solutions of (6.1) are solutions of (6.2), i.e.,

$$
\begin{array}{r}
\tilde{P}\{(y, \omega) \text { : there exists a solution } x \text { of }(6.1) \text { such that } \dot{x}(t) \notin \exp F(x(t)) \\
\text { in a t-set of positive Lebesgue measure }\}=0 .
\end{array}
$$

Moreover, with probability one the sequence constructed according to (2.7) is relatively compact in the strong topology of $W^{1,1}\left([0, T] ; \mathbb{R}^{2}\right)$. 
Proof. Fix $T>0$. Given an arbitrary sample path $\theta=\theta^{y, \omega} \in \Theta$ define $X^{\theta}$ to be the set of all solutions to (6.1) and observe that $X^{\theta}$ is compact in $\mathcal{C}\left([0, T] ; \mathbb{R}^{2}\right)$. Applying properties of measurable relations (see, e.g., [10]) one can easily prove that the map $\theta \mapsto X^{\theta}$ is measurable. Therefore, by the separability of the space $\mathcal{C}\left([0, T] ; \mathbb{R}^{2}\right)$, there exists a sequence of measurable selections $\left\{x_{n}^{\theta}\right\}$ such that $X^{\theta}$ equals the closure of $\left\{x_{n}^{\theta}\right\}$ in $\mathcal{C}\left([0, T] ; \mathbb{R}^{2}\right)$ for all $\theta$. We wish to prove that, almost surely,

$$
\max _{x \in X^{\theta}} \operatorname{diam}\left(F^{\nu_{\theta}(t)}(x(t))\right)>0
$$

on a $t$-set of Lebesgue measure zero. Here diam $(A)$ means the diameter of a set $A$. Observe that, thanks to the upper semicontinuity of the map $x \mapsto F^{\nu_{\theta}(t)}(x)$, for all $t \in[0, T]$ the maximum above is attained and equals the supremum over the sequence $\left\{x_{n}^{\theta}\right\}$. Thus we need to show that, for every $n \in \mathbb{N}$,

$$
\begin{aligned}
Q \otimes P\{\theta: \exists E & =E(\theta) \text { with }|E|>0 \text { such that } \\
& \left.\operatorname{diam}\left(F^{\nu_{\theta}(t)}\left(x_{n}^{\theta}(t)\right)\right)>0 \forall t \in E\right\}=0 .
\end{aligned}
$$

To this aim, fix $n$ and consider the function (we now drop the subscript $n$ from $x_{n}^{\theta}$ )

$$
\Psi(\theta, t)=\operatorname{diam}\left(F^{\nu_{\theta}(t)}\left(x^{\theta}(t)\right)\right),
$$

which is jointly measurable (see [10]).

Fix $\eta>0$ and consider the measurable sets

$$
E_{\eta}(\theta):=\{t \in[0, T]: \Psi(\theta, t)>\eta\}, \quad \theta \in \Theta,
$$

and

$$
\Theta_{\eta}:=\left\{\theta:\left|E_{\eta}(\theta)\right|>0\right\} .
$$

Observe that $\theta \in \Theta_{\eta}$ means that

$$
\Gamma(\theta, t):=F^{\nu_{\theta}(t)}\left(x^{\theta}(t)\right)
$$

is a segment in $\mathbb{R}^{2}$ of length greater than $\eta$ on a set of positive measure. Our goal now is to prove that $Q \otimes P\left(\Theta_{\eta}\right)=0$. To this end we fix $\theta \in \Theta_{\eta}$ and choose a closed set $\hat{E}_{\eta}(\theta) \subset E_{\eta}(\theta)$ with positive measure such that the mappings $t \mapsto \Gamma(\theta, t), t \mapsto \Psi(\theta, t)$ and $t \mapsto \nu_{\theta}(t)$ restricted to $\hat{E}_{\eta}(\theta)$ are continuous. Without loss of generality we can assume that each point of $\hat{E}_{\eta}(\theta)$ is of positive density with respect to $\hat{E}_{\eta}(\theta)$.

Fix $\bar{t} \in \hat{E}_{\eta}(\theta)$ and given $0<\varepsilon<\eta / 3$ choose $\delta=\delta(\theta)>0$ such that

$$
\begin{aligned}
\left|\nu_{\theta}(t)-\nu_{\theta}(\bar{t})\right| & \leq \frac{\pi}{4}, \\
H(\Gamma(\theta, t), \Gamma(\theta, \bar{t})) & \leq \frac{\varepsilon}{3},
\end{aligned}
$$

and

$$
|\Psi(\theta, t)-\Psi(\theta, \bar{t})| \leq \frac{\varepsilon}{3}
$$

whenever $t \in \hat{E}_{\eta}(\theta)$ with $|t-\bar{t}| \leq \delta$. We can represent $\Gamma(\theta, \bar{t})$ as $\Gamma(\theta, \bar{t})=$ $\{\lambda \bar{a}+(1-\lambda) \bar{b}: \lambda \in[0,1]\}$ for some $\bar{a}, \bar{b} \in \mathbb{R}^{2}$ with $|\bar{a}-\bar{b}|>\eta$, and observe 
that the set $\tilde{E}_{\eta}(\theta):=\hat{E}_{\eta}(\theta) \cap[\bar{t}-\delta, \bar{t}+\delta]$ has positive measure as well. We now use Lemma 3.1, which gives

$$
\begin{aligned}
\sup _{p \in S^{1}}\left|\sigma_{F\left(x^{\theta}(t)\right)}(p)-\sigma_{F\left(x^{\theta}(s)\right)}(p)\right| & =H\left(F\left(x^{\theta}(t)\right), F\left(x^{\theta}(s)\right)\right) \\
& \leq L\left|x^{\theta}(t)-x^{\theta}(s)\right|^{\alpha} \\
& \leq L M^{\alpha}|t-s|^{\alpha}
\end{aligned}
$$

for all $s, t \in \tilde{E}_{\eta}(\theta)$ (recall that $F\left(x^{\theta}(t)\right) \subset B(0, M)$ ). For each $t \in \tilde{E}_{\eta}(\theta)$ choose an arbitrary point $a_{t} \in \Gamma(\theta, t)$. Taking into account that $\nu_{\theta}(t) \in S^{1}$ and

$$
\nu_{\theta}(t) \cdot a_{t}=\sigma_{F\left(x^{\theta}(t)\right)}\left(\nu_{\theta}(t)\right),
$$

we deduce from (6.4) that

$$
\nu_{\theta}(s) \cdot\left(a_{t}-a_{s}\right) \leq L M^{\alpha}|t-s|^{\alpha}
$$

and

$$
\nu_{\theta}(t) \cdot\left(a_{s}-a_{t}\right) \leq L M^{\alpha}|t-s|^{\alpha} .
$$

By summing the inequalities (6.5) and (6.6) we obtain

$$
\left(\nu_{\theta}(t)-\nu_{\theta}(s)\right) \cdot\left(a_{s}-a_{t}\right) \leq 2 L M^{\alpha}|t-s|^{\alpha} .
$$

It follows from simple geometric considerations that for each $t \in \tilde{E}_{\eta}(\theta)$ the endpoints of the segment $\Gamma(\theta, t)$ are located in the $\varepsilon$-neighborhoods of the points $\bar{a}$ and $\bar{b}$, respectively. Consequently, for every $s, t \in \tilde{E}_{\eta}(\theta)$ we can choose suitable $a_{t} \in \Gamma(\theta, t) \cap B(\bar{a}, \varepsilon), a_{s} \in \Gamma(\theta, s) \cap B(\bar{b}, \varepsilon)$ or $a_{t} \in \Gamma(\theta, t) \cap B(\bar{b}, \varepsilon)$, $a_{s} \in \Gamma(\theta, s) \cap B(\bar{a}, \varepsilon)$ such that the scalar product in (6.7) is positive. On the other hand, since $\left\|\nu_{\theta}(t)\right\|=\left\|\nu_{\theta}(s)\right\|=1$ elementary geometric considerations show that $\nu_{\theta}(t)-\nu_{\theta}(s)$ is parallel to some vector with endpoints in $B(\bar{a}, \varepsilon)$ and $B(\bar{b}, \varepsilon)$. Therefore we conclude that

$$
\sin \gamma(t, s) \leq \frac{2 \varepsilon}{\eta}
$$

where $\gamma(t, s)$ is the angle between the vectors $\nu_{\theta}(t)-\nu_{\theta}(s)$ and $a_{s}-a_{t}$. Combining the inequalities (6.7) and (6.8) we obtain

$$
\left\|\nu_{\theta}(t)-\nu_{\theta}(s)\right\|\left\|a_{t}-a_{s}\right\| \leq \frac{2 L M^{\alpha} \eta}{\sqrt{\eta^{2}-4 \varepsilon^{2}}}|t-s|^{\alpha} .
$$

Since $\left\|a_{t}-a_{s}\right\| \geq \Psi(\theta, \bar{t})-2 \varepsilon>\eta-2 \varepsilon \geq \eta / 3$, we finally obtain from (6.9)

$$
\left\|\nu_{\theta}(t)-\nu_{\theta}(s)\right\| \leq \frac{18 L M^{\alpha}}{\sqrt{5} \eta}|t-s|^{\alpha}, \quad t, s \in \tilde{E}_{\eta}(\theta) .
$$

Recalling Theorem 4.1 and (6.10), we obtain

$$
Q \otimes P\left(\Theta_{\eta}\right)=\frac{1}{2 \pi} \int_{0}^{2 \pi} P\left\{\Theta_{\eta} \mid Y=y\right\} d y=0 .
$$


Setting $\eta=\frac{1}{n}, n=1,2, \ldots$, we see that $\left\{\Theta_{1 / n}\right\}$ is an increasing sequence of measurable subsets of $\Theta$ such that

$$
\Theta_{0}:=\{\theta \in \Theta:|\{t: \Psi(\theta, t)>0\}|>0\}=\bigcup_{n=1}^{\infty} \Theta_{1 / n},
$$

and $Q \otimes P\left(\Theta_{0}\right)=0$. Thus (6.3) is proved.

The statement on the strong compactness follows from Theorem 4.1 in [6] (see also the proof of Lemma 3.2 in the same paper) or from [15, Theorem 4.1], by taking into account that exposed points are also extremal. However, for the sake of completeness we give here a direct proof using exposedness.

Since the sequence of solutions to $(2.7)$ is relatively weakly compact in $W^{1,1}\left([0, T] ; \mathbb{R}^{2}\right)$, it suffices to prove that each sequence of solutions $\left\{x_{n}(\cdot)\right\}$ to $(2.1)-(2.2)$ weakly converging in $W^{1,1}\left([0, T] ; \mathbb{R}^{2}\right)$ to an exposed solution $x(\cdot)$ converges also strongly, i.e.,

$$
\int_{0}^{T}\left\|\dot{x}_{n}(t)-\dot{x}(t)\right\| d t \rightarrow 0 \quad \text { as } \quad n \rightarrow \infty .
$$

First off all, by the measurable selection theorem we can associate with $\left\{\dot{x}_{n}(\cdot)\right\}$ a sequence of measurable functions $v_{n}(t) \in F(x(t))$ such that

$$
\left\|\dot{x}_{n}(t)-v_{n}(t)\right\| \leq L\left\|x_{n}(t)-x(t)\right\|^{\alpha} \rightarrow 0,
$$

$t \in[0, T]$. The convergence in (6.12) follows from the weak convergence of the derivatives $\dot{x}_{n}(\cdot)$ in $L_{1}\left([0, T] ; \mathbb{R}^{2}\right)$. Hence, the sequence $\left\{v_{n}(\cdot)\right\}$ converges to $\dot{x}(\cdot)$ weakly as well. Since $\dot{x}(t) \in \exp F(x(t))$ for a.e. $t \in[0, T]$, there exists a measurable function $p(\cdot),\|p(t)\|=1$, such that

$$
p(t) \cdot(\dot{x}(t)-y)>0
$$

for all $y \in F(x(t))$ with $y \neq \dot{x}(t)$. Assuming that $\left\{v_{n}(\cdot)\right\}$ does not converge to $\dot{x}(\cdot)$ in measure, we choose $\varepsilon>0$ and $\delta>0$ such that, up to a subsequence,

$$
\left|\left\{t \in[0, T]:\left\|v_{n}(t)-\dot{x}(t)\right\| \geq \varepsilon\right\}\right| \geq \delta, n \geq 1 .
$$

Let us consider the measurable function (modulus of rotundity)

$$
\rho_{\varepsilon}(t):=\inf \{p(t) \cdot(\dot{x}(t)-y): y \in F(x(t)),\|\dot{x}(t)-y\| \geq \varepsilon\}, t \in[0, T],
$$

and find a closed set $\mathcal{T} \subset[0, T]$ such that $|[0, T] \backslash \mathcal{T}| \leq \delta / 2$ and $\rho_{\varepsilon}(t)$ is continuous on $\mathcal{T}$. It follows from (6.13) that

$$
m:=\min _{t \in \mathcal{T}} \rho_{\varepsilon}(t)>0 .
$$

Denoting by $\mathcal{T}_{n}$ the measurable set in (6.14), observe that $\left|\mathcal{T}_{n} \cap \mathcal{T}\right| \geq \delta / 2$. On the other hand, for $t \in \mathcal{T}_{n}$,

$$
p(t) \cdot\left(\dot{x}(t)-v_{n}(t)\right) \geq \rho_{\varepsilon}(t) \geq m 1_{\mathcal{T}_{n} \cap \mathcal{T}}(t) .
$$

Integrating the left-hand side of (6.15) and using (6.13) and (6.15), we obtain

$$
\int_{0}^{T} p(t) \cdot\left(\dot{x}(t)-v_{n}(t)\right) d t \geq m\left|\mathcal{T}_{n} \cap \mathcal{T}\right| \geq \frac{m \delta}{2}>0
$$


which contradicts the weak convergence. Thus, recalling (6.12) and using the convergence in measure proved above we obtain (6.11).

Remarks. 1. It is reasonable to conjecture that the above result can be generalized to $N$-dimensional spaces, provided $\partial F$ admits only extremal faces of dimension 0 and $N-1$.

2. In the above setting we are not able to define a measure $\mu$ on $\mathcal{C}([0, T]$; $\left.\mathbb{R}^{2}\right)$ with the properties $\left(P_{1}\right)$ and $\left(P_{2}\right)$ stated in Sect. 2 . In fact, although the proof shows that the differential inclusion (2.5) (with $\theta(t)$ in place of $\omega(t)$ in the present notation) is a.s. a differential equation, we are not able to prove uniqueness of solutions, even if $F$ is Lipschitz. This fact prevents the use of the push-forward of $Q \otimes P$.

\section{The $x$ independent case}

This section is devoted to the much easier case where the space variable in the right-hand side $F$ of (2.1) is replaced by the time variable. All results can be proved here in full generality.

We recall first a result on convex sets.

Proposition 7.1. Let $K \subset \mathbb{R}^{N}$ be compact and convex. Then, for $\mathcal{H}^{N-1}$-a.e. $p \in$ $S^{N-1}$, the problem

$$
\max _{y \in K} p \cdot y
$$

admits a unique solution.

Proof. It is enough to observe that the support function $\sigma_{K}(p)$ is $\mathcal{H}^{N-1}$-a.e. differentiable on $S^{N-1}$ by Rademacher's theorem and to recall the representation (3.2).

Let $F$ be a measurable map from $[0, T]$ into the compact and convex subsets of $\mathbb{R}^{N}$ and let $(\Omega, P)$ denote Brownian motion on $S^{N-1}$ whose sample paths $\omega(t)$ are uniformly distributed on $S^{N-1}$. For every $\omega \in \Omega$, we define (see (2.4))

$$
F^{\omega}(t)=F^{\omega(t)}(t) \text {. }
$$

Then $(t, \omega) \mapsto F^{\omega}(t)$ is jointly measurable (see [10]) and, thanks to Proposition 7.1, $P$-a.s. $F^{\omega}(t)$ is a singleton for a.e $t \in[0, T]$. Set, for all $\omega \in \Omega, t \in[0, T]$,

$$
x^{\omega}(t)=\bar{x}+\int_{0}^{t} F^{\omega}(s) d s,
$$

where the integral is meant in the Aumann sense if $F^{\omega}$ is not single valued, and

$$
\mathcal{S}=\left\{x^{\omega}(\cdot): \omega \in \Omega\right\}, \quad \mathcal{S}^{\exp }=\left\{x^{\omega}(\cdot): \omega \in \Omega, F^{\omega}(\cdot) \text { is a.e. a singleton }\right\} .
$$

Define the probability measure $\mu$ on $\mathcal{C}\left([0, T] ; \mathbb{R}^{N}\right)$ by setting, for each Borel set $B \subset \mathcal{C}\left([0, T] ; \mathbb{R}^{N}\right)$,

$$
\mu(B)=P\left\{\omega: x^{\omega} \text { is a singleton and } x^{\omega} \in B\right\} .
$$

Then we have 
Theorem 7.2. Under the above assumptions on $F$ and $\Omega$, the following holds:

1. $\mu\left(\mathcal{S}^{\exp }\right)=1$;

2. the support of $\mu$ is $\mathcal{S}$;

3. P-a.s. on $\Omega$, the sequence $\left\{x_{m}^{\omega}\right\}$ defined according to (2.7) converges to $x^{\omega}$ strongly in $W^{1,1}\left(0, T ; \mathbb{R}^{N}\right)$.

Proof. For the sake of brevity, the proof will only be sketched.

Statement 1) is obvious by construction. Statement 2) can be proved with the same argument of part 1) in Proposition 5.3, by using Lyapunov's Convexity Theorem and Proposition 4.3. More precisely, let $x(t)=\bar{x}+\int_{0}^{t} \xi(s) d s$, $\xi(s) \in F^{\omega}(s), s \in[0, T]$, be in $\mathcal{S}$. Recalling Theorem 18.7 in [14] and [10], there exist sequences $\left\{\xi_{n}^{j}: n \in \mathbb{N}\right\}$ of measurable selections of $t \mapsto \exp F(t)$ and of measurable functions $\left\{\lambda_{n}^{j}\right\}, n \in \mathbb{N}, j=1, \ldots, N+1$ with values in $[0,1]$ such that $\sum_{j=1}^{N+1} \lambda_{n}^{j} \equiv 1$ for all $n$ and

$$
\xi(t)=\lim _{n \rightarrow \infty} \sum_{j=1}^{N+1} \lambda_{n}^{j}(t) \xi_{n}^{j}(t) \quad \text { for a.e. } t \in[0, T] .
$$

Partition $[0, T]$ into subintervals $I_{m}^{i}$ of length $T / m, m=1,2, \ldots, i=1, \ldots, m$. By Lyapunov's Convexity Theorem (see [12, Theorem 2]) for each $n \in \mathbb{N}$ there exist measurable partitions $E_{m, n}^{i, j}$ of the intervals $I_{m}^{i}$ such that, for all $n \in \mathbb{N}$, $m=1,2, \ldots, j=1, \ldots, N+1, i=1, \ldots, m$ one has

$$
\int_{E_{m, n}^{i, j}} \xi_{n}^{j}(t) d t=\int_{I_{m}^{i}} \lambda_{n}^{j}(t) \xi_{n}^{j}(t) d t
$$

Define, for $n \in \mathbb{N}, t \in[0, T]$,

$$
u_{m, n}(t)=\sum_{i=1}^{m} \sum_{j=1}^{N+1} 1_{E_{m, n}^{i, j}}(t) \xi_{n}^{j}(t), \quad x_{m, n}(t)=\bar{x}+\int_{0}^{t} u_{m, n}(t) d t .
$$

Now it is easy to see that the sequence $\left\{x_{n, n}\right\}$ converges to $x$ uniformly in $[0, T]$. The remainder of the proof goes on exactly as in Proposition 5.3. Statement 3) follows from the fact that $P$-a.s. $\dot{x}^{\omega}(t) \in \exp F(t)$ for a.e. $t \in[0, T]$ by using Olech's Lemma (see Lemma 1 in [12]), or the same argument presented at the end of the proof of Theorem 6.1.

\section{Acknowledgments}

The authors wish to express their gratitude to A. Bressan for kindly suggesting the problem and the outline of the proof for the case of a segment. They are also deeply indebted with P. Dai Pra for suggesting a crucial part of the argument leading to Theorem 4.1 and for several discussions on probability issues. 


\section{Appendix: the modulus of continuity of an auxiliary map}

The section is devoted to the following result on the modulus of continuity of the map defined by formula (2.6). Observe that the problem considered here does not fit into the standard results for intersections of set valued maps, since the values of $F$ may have empty interior.

Proposition 8.1. Let $F$ be a Hausdorff continuous map from $\mathbb{R}^{N}$ into the family of all compact and convex subsets of $\mathbb{R}^{N}$. Assume that $F$ is bounded, i.e., there exists $M$ such that $\|v\| \leq M$ for all $v \in F(x), x \in \mathbb{R}^{N}$. Fix $\varepsilon>0$ and define

$$
G_{\varepsilon}(x, p)=\left\{v \in F(x): \sigma_{F(x)}(p) \leq v \cdot p+\varepsilon\right\},
$$

$x \in \mathbb{R}^{N}, p \in S^{N-1}$. Then, there exists a constant $C$ independent of $M, \varepsilon$ such that, for all $x^{\prime}, x^{\prime \prime} \in \mathbb{R}^{N}$ and all $p \in S^{N-1}$,

$$
H\left(G_{\varepsilon}\left(x^{\prime}, p\right), G_{\varepsilon}\left(x^{\prime \prime}, p\right)\right) \leq \frac{C M}{\varepsilon} H\left(F\left(x^{\prime}\right), F\left(x^{\prime \prime}\right)\right) .
$$

Proof. We use here the representation of the Hausdorff distance given by Lemma 3.1.

Fix $x \in \mathbb{R}^{N}$. We claim that for each $p, q \in S^{N-1}$

$$
\sigma_{G_{\varepsilon}(x, p)}(q)=\inf _{\lambda \geq 0}\left[\sigma_{F(x)}(q+\lambda p)+\lambda\left(\varepsilon-\sigma_{F(x)}(p)\right) \vee 0\right],
$$

where the infimum is attained at some $0 \leq \bar{\lambda} \leq 2 M / \epsilon$.

In order to prove the above claim, set $A=F(x)$ and consider the closed affine half space

$$
\Delta(A, p)=\left\{v \in \mathbb{R}^{N}: \sigma_{A}(p) \leq \varepsilon+v \cdot p\right\} .
$$

Observe that $A \cap \operatorname{int} \Delta(A, p) \neq \emptyset$, so that $0 \in \operatorname{int}(A-\Delta(A, p))$. Therefore we can apply $[2,(31)$, p. 32], obtaining that

$$
\sigma_{G_{\varepsilon}(x, p)}(q)=\inf _{q_{1}+q_{2}=q}\left[\sigma_{A}\left(q_{1}\right)+\sigma_{\Delta(A, p)}\left(q_{2}\right)\right],
$$

where the infimum is taken among those $q_{2}$ for which $\sigma_{\Delta(A, p)}\left(q_{2}\right)<+\infty$. Obviously, we have

$$
\sigma_{\Delta(A, p)}\left(q_{2}\right)= \begin{cases}\lambda\left(\varepsilon-\sigma_{A}(p)\right) & \text { if } q_{2}=-\lambda p, \sigma_{A}(p) \leq \varepsilon, \lambda \geq 0 \\ 0 & \text { if } q_{2}=-\lambda p, \sigma_{A}(p)>\varepsilon, \lambda \geq 0 \\ +\infty & \text { otherwise. }\end{cases}
$$

Therefore,

$$
\sigma_{G_{\varepsilon}(A, p)}(q)=\inf _{\lambda \geq 0}\left[\sigma_{A}(q+\lambda p)+\lambda\left(\varepsilon-\sigma_{A}(p)\right) \vee 0\right]
$$

Set

$$
\psi(\lambda)=\sigma_{A}(q+\lambda p)+\lambda\left(\varepsilon-\sigma_{A}(p)\right) \vee 0
$$

and observe that $\psi(\lambda) \geq \lambda \varepsilon-\sigma_{A}(-q)$ for all $\lambda \geq 0$ and all $p, q$. Thus, being continuous and convex with respect to $\lambda$, this function admits a minimizer $\bar{\lambda}=\bar{\lambda}(A, p, q) \geq 0$. Obviously, $\sigma_{G_{\varepsilon}(A, p)}(q)=\psi(\bar{\lambda}) \leq \psi(0)=\sigma_{A}(q)$. Set $\lambda^{*}=$ $\left(\sigma_{A}(q)+\sigma_{A}(-q)\right) / \varepsilon$ and observe that necessarily we have $\psi\left(\lambda^{*}\right) \geq \lambda^{*} \varepsilon-$ 
$\sigma_{A}(-q)=\sigma_{A}(q)$. Moreover, $\lambda^{*} \geq 0$ and $\lambda^{*}=0$ if and only if $q \cdot y$ is constant over $y \in A$, whence $\bar{\lambda}=0$. Therefore

$$
\bar{\lambda} \leq \lambda^{*} \leq \frac{\sigma_{A}(q)+\sigma_{A}(-q)}{\varepsilon} \leq \frac{2 M}{\varepsilon}
$$

as claimed.

We are now ready to conclude the proof of the theorem. To this aim, fix $q \in S^{N-1}$ and let $x^{\prime}, x^{\prime \prime} \in \mathbb{R}^{N}, p \in S^{N-1}$. Assume first $\sigma_{F\left(x^{\prime \prime}\right)}(q) \leq \varepsilon$ and let $\bar{\lambda}$ be taken from the above claim for $A=F\left(x^{\prime \prime}\right)$. Then by (8.1) we have

$$
\begin{aligned}
\sigma_{G_{\varepsilon}\left(x^{\prime}, p\right)}(q) \geq & \sigma_{F\left(x^{\prime \prime}\right)}(q+\bar{\lambda} p)+\bar{\lambda}\left(\varepsilon-\sigma_{F\left(x^{\prime \prime}\right)}(p)\right) \\
& \quad-\left|\sigma_{F\left(x^{\prime \prime}\right)}(q+\bar{\lambda} p)-\sigma_{F\left(x^{\prime}\right)}(q+\bar{\lambda} p)\right| \\
& \quad-\bar{\lambda}\left|\sigma_{F\left(x^{\prime \prime}\right)}(p)-\sigma_{F\left(x^{\prime}\right)}(p)\right| \\
\geq & \sigma_{G_{\varepsilon}\left(x^{\prime \prime}, p\right)}(q)-(\|q+\bar{\lambda} p\|+\bar{\lambda}) H\left(F\left(x^{\prime}\right), F\left(x^{\prime \prime}\right)\right) \\
\geq & \sigma_{G_{\varepsilon}\left(x^{\prime \prime}, p\right)}(q)-\left(\frac{2 M}{\varepsilon}+\sqrt{1+\frac{4 M}{\varepsilon}+\frac{4 M^{2}}{\varepsilon^{2}}}\right) H\left(F\left(x^{\prime}\right), F\left(x^{\prime \prime}\right)\right) .
\end{aligned}
$$

The case $\sigma_{F\left(x^{\prime \prime}\right)}(q)>\varepsilon$ is easier and can be handled similarly. The proof is concluded since we can interchange the role of $x^{\prime}$ and $x^{\prime \prime}$.

\section{References}

[1] Aubin, J.P., Cellina, A.: Differential Inclusions. Springer, Berlin (1984)

[2] Aubin, J.P., Ekeland, I.: Applied Nonlinear Analysis. Wiley, New York (1984)

[3] Bressan, A., Colombo, G.: Generalized Baire category and differential inclusions in Banach spaces. J. Differ. Equ. 76, 135-158 (1988)

[4] Cellina, A.: On the differential inclusion $x^{\prime} \in[-1,1]$. Atti Accad. Naz. Lincei Rend. Cl. Sci. Fis. Mat. Natur. 69, 1-6 (1981)

[5] Cellina, A.: A view on differential inclusions. Rend. Semin. Mat. Univ. Politec. Torino 63, 197-209 (2005)

[6] Colombo, G.: On extremal solutions of differential inclusions. Bull. Polish Acad. Sci. Math. 40, 97-109 (1992)

[7] De Blasi, F.S., Pianigiani, G.: On the Baire category method in existence problems for ordinary and partial differential inclusions. In: Set Valued Mappings with Applications in Nonlinear Analysis, pp. 137-148. Taylor \& Francis, London (2002)

[8] De Blasi, F.S., Pianigiani, G.: Baire category and the weak bang-bang property for continuous differential inclusions. Proc. Am. Math. Soc. 138, 24132423 (2010)

[9] Filippov, A.F.: On the existence of solutions of multivalued differential equations. Mat. Zametki 10, 307-313 (1970) 
[10] Himmelberg, C.J.: Measurable relations. Fund. Math. LXXXVII 52-72 (1975)

[11] Karatzas, I., Shreve, S.E.: Brownian Motion and Stochastic Calculus. Springer, New York (1988)

[12] Olech, Cz.: The Lyapunov theorem: its extensions and applications. In: Cellina, A. (ed.) Proc. C.I.M.E., Varenna. Lecture Notes in Math., vol. 1446. Springer, Berlin (1990)

[13] Pianigiani, G.: Differential inclusions. The Baire category method. In: Cellina, A. (ed.) Proc. C.I.M.E., Varenna. Lecture Notes in Math., vol. 1446. Springer, Berlin (1990)

[14] Rockafellar, R.T.: Convex Analysis. Princeton University Press, Princeton (1970)

[15] Rzeżuchowski, T.: Strong convergence of selections implied by weak. Bull. Austral. Math. Soc. 39, 201-214 (1989)

[16] Walter, W.: Ordinary Differential Equations. Springer, New York (1998)

Giovanni Colombo

Dipartimento di Matematica Pura ed Applicata

Università di Padova

via Trieste 63, 35121 Padua

Italy

e-mail: colombo@math.unipd.it

Vladimir V. Goncharov

Departamento de Matemática

Universidade de Évora

Colégio Luís António Verney

Rua Romão Ramalho, 59

7000-671 Évora, Portugal

e-mail: goncha@uevora.pt

Received: 24 January 2012.

Accepted: 26 March 2012. 